\title{
Restoration Melanin of Fonseace monophora reduced Its Pathogenicity to Galleria mellonella
}

Yinghui Liu, ${ }^{1,2}$ Liyan Xi, ${ }^{1}$ and Chester R. Cooper Jr${ }^{2}$

1. Dermatology Hospital, Southern Medical University

2. Biological Sciences, Youngstown State University

\section{Introduction}

Galleria mellonella has been widely used as a heterologous host for fungal pathogens. Melanin has been linked to Fonsecaea monophora virulence. while its role and mechanisms remains unclear. Herein we address this question in an Galleria mellonella infection model using albino and melanin strains of Fonsecaea monophora .

\section{Methods:}

1. Restore the melanin of albino mutant of $F$. monophora by adding exogenous scytalone released by the mell mutant of $E$. dermatitidis.

2. Galleria mellonella infection model were established with albino and melanin strain of $F$. monophora, then the fungal virulence, histopathology were compared.

\section{$\underline{\text { Results: }}$}

Albino mutant displayed a higher level of pathogenicity than melanized strain, and restoration its melanin can reverse it.

1

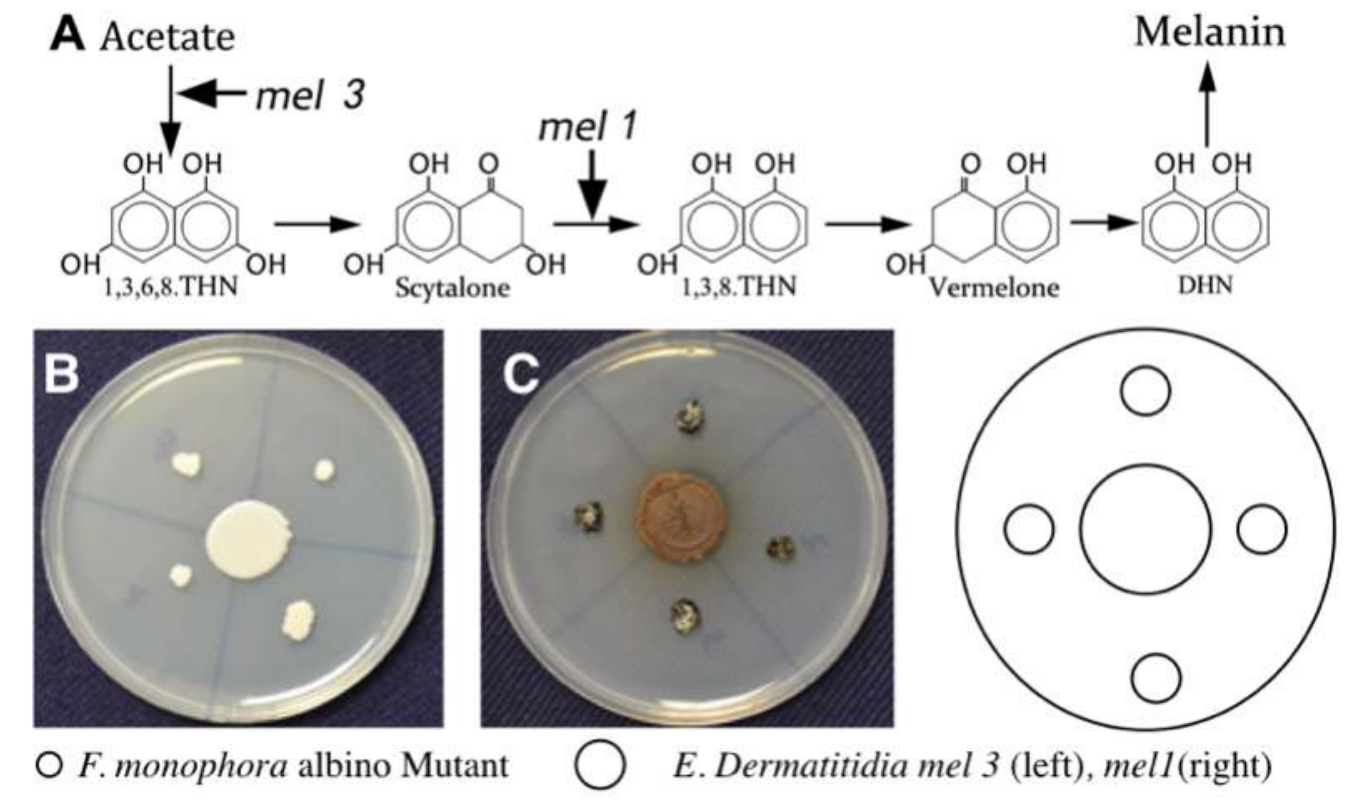

Fig1.Melanin of albino mutant can be restored by E. dermatitidia

2
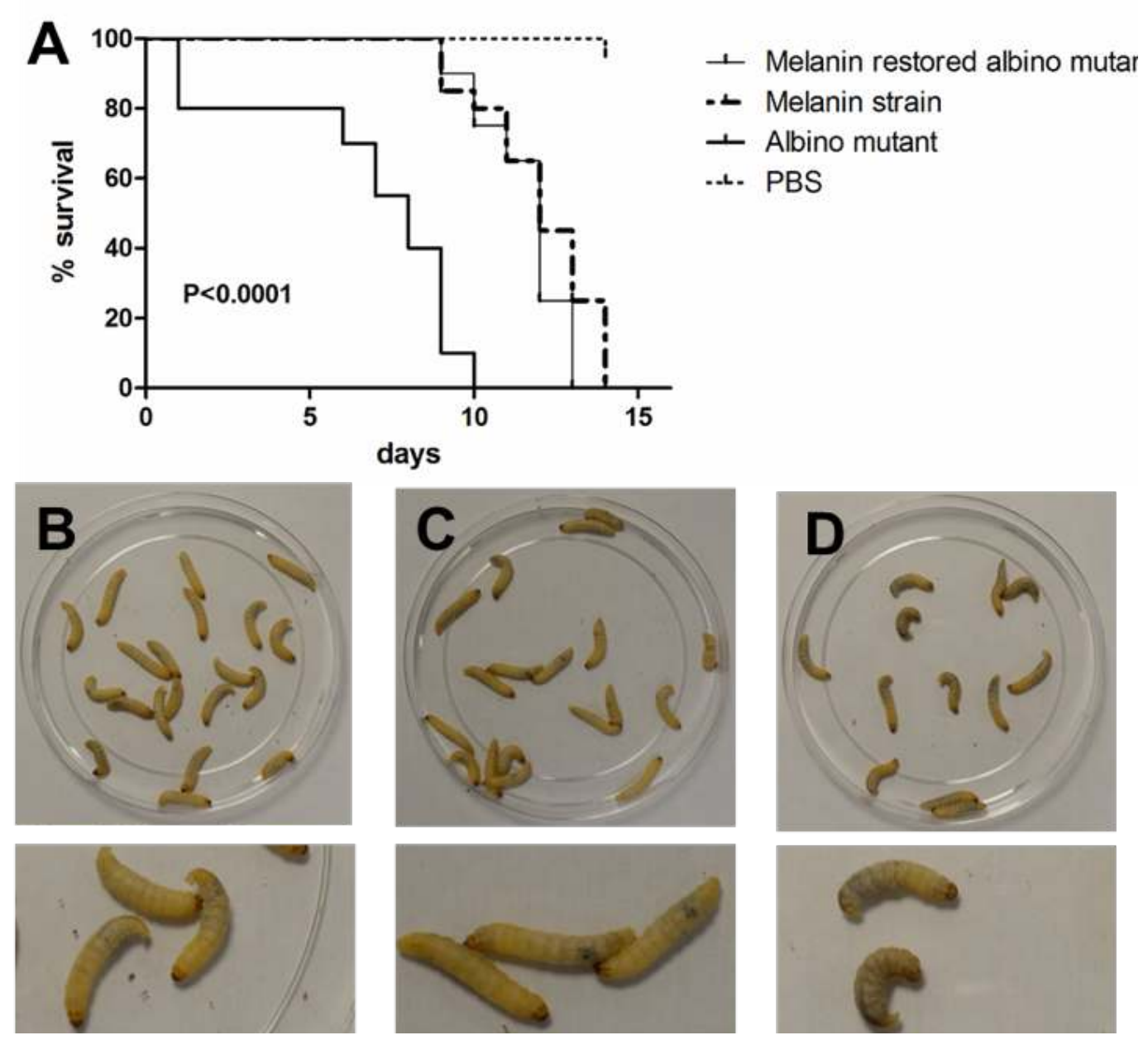

Fig2. (A) Survival curves of Galleria mellonella.

Infection with $F$. monophora albino mutant causes $G$. mellonella larvae to darken after inoculation (3 days). Larvae were infected with $F$ monophora in which melanin was restored in the albino mutant (A), melanized strain (B), and albino mutant $(\mathrm{C})$.

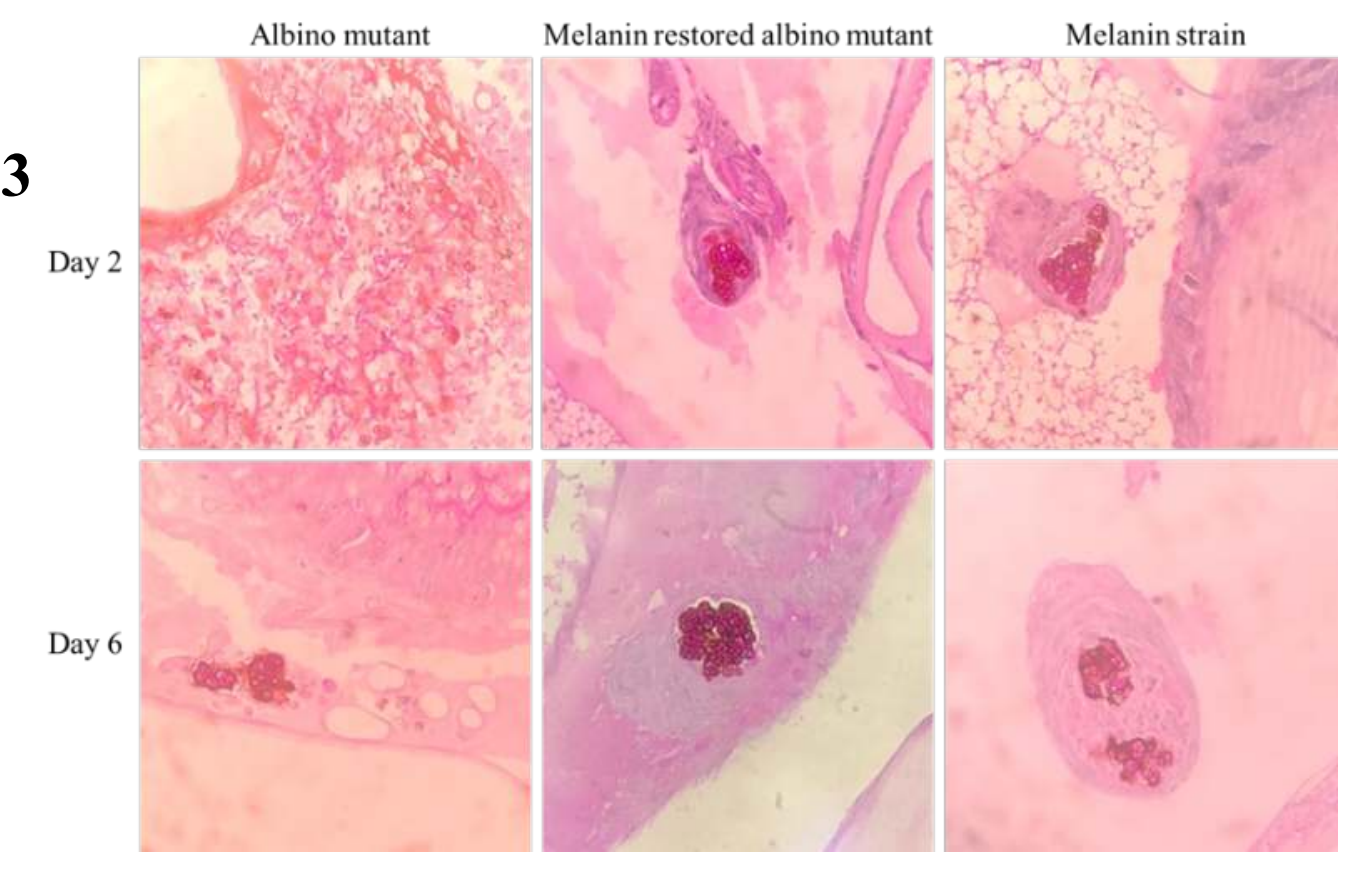

Fig 3 Histopathology of $G$ mellonella infected with the albino mutant, melanin restored strain and melanin strain: Melanin may activate larvae immune response and promote the formation of inflammatory nodules 。

\section{Supplement study of F.monophora melanin}

Melanin of F.monophora Modulates Murine Macrophage polarization

4

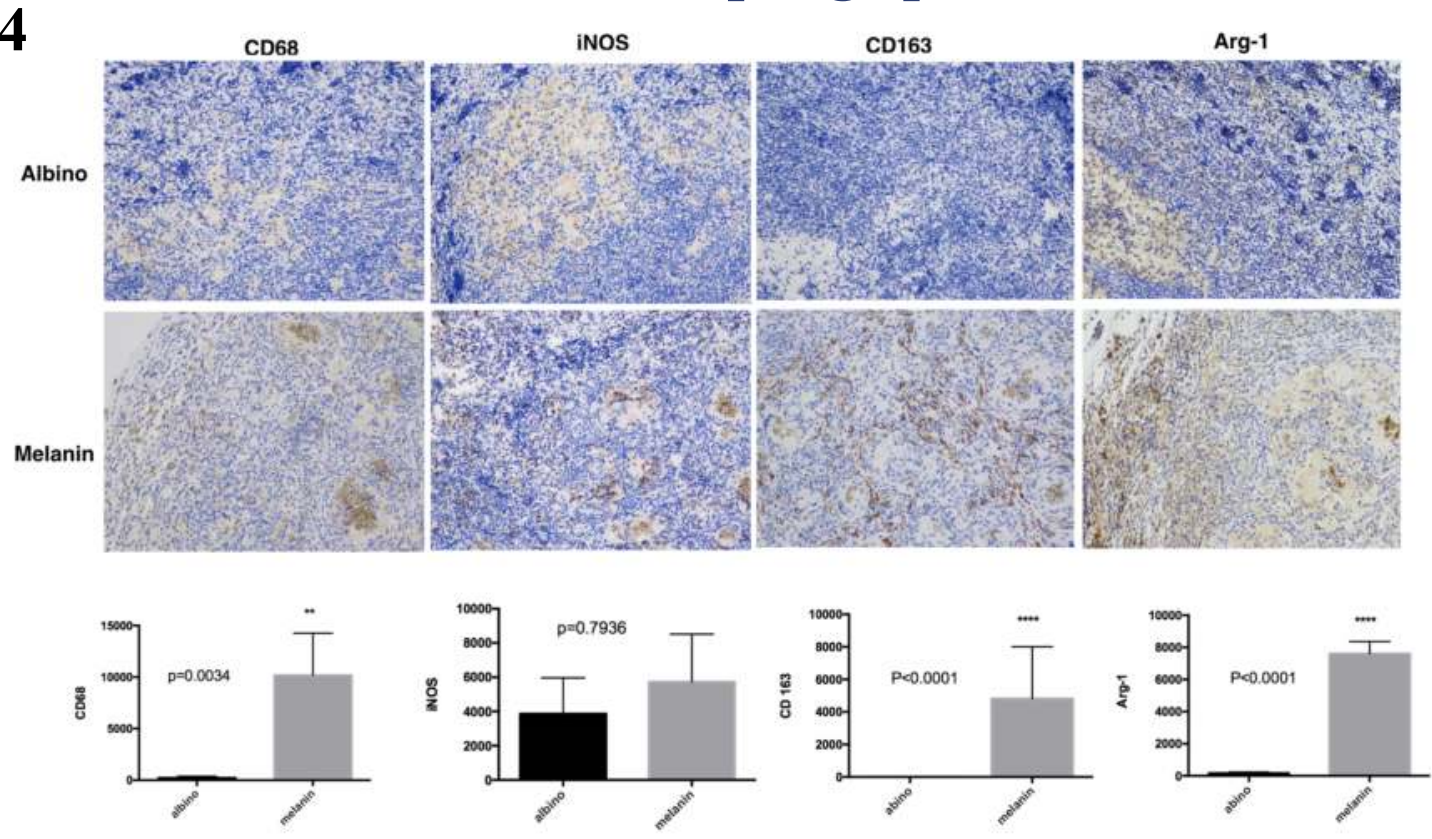

Fig.4 F. monophora melanin could alter Murine macrophage phenotype to M2,features a decreased expression of M1 marker iNOS and increased expression of M2 marker Arg-1.

\section{Discussions}

Fungal melanin has been shown to be important for virulence in mammal models for several pathogenic fungi. However, the results from the current study demonstrate that the lack of melanin enhances $F$. monophora virulence in the insect $G$. mellonella. Similar phenomenon might also exist for different species of the pathogenic fungi Cryptococcus and Aspergillus fumigatus. Previously, it is believed that the exacerbated immune response of $G$. mellonella induced by increased exposure of antigens normally hidden by melanin may cause self-damage may result in increased mortality of larvae infected with the albino mutant. However, Histopathology revealed that melanin can attract immune cells and promote the formation of inflammatory nodules. Further study of F.monophora melanin in Murine model showed that melanin could alter macrophage phenotype to M2. Possibly, melanin could control self-damage of infected larvae by alter macrophage phenotype to M2 which have anti-inflammatory Effects. Our hypothesis is credible based on previous studies that immune responses can cause selfdamage and that melanin contributes to modulate host macrophage to $\mathrm{M} 2$. 